\title{
BMJ Open Validity of peptic ulcer disease and upper gastrointestinal bleeding diagnoses in administrative databases: a systematic review protocol
}

\author{
Alessandro Montedori, ${ }^{1}$ Iosief Abraha, ${ }^{1}$ Carlos Chiatti, ${ }^{2}$ Francesco Cozzolino, ${ }^{1}$ \\ Massimiliano Orso, ${ }^{1}$ Maria Laura Luchetta, ${ }^{3}$ Joseph M Rimland, ${ }^{4}$ \\ Giuseppe Ambrosio ${ }^{5}$
}

To cite: Montedori A, Abraha I, Chiatti C, et al. Validity of peptic ulcer disease and upper gastrointestinal bleeding diagnoses in administrative databases: a systematic review protocol. BMJ Open 2016;6:e011776.

doi:10.1136/bmjopen-2016011776

- Prepublication history and additional material is available. To view please visit the journal (http://dx.doi.org/ 10.1136/bmjopen-2016011776).

Received 4 March 2016 Revised 16 June 2016 Accepted 20 June 2016

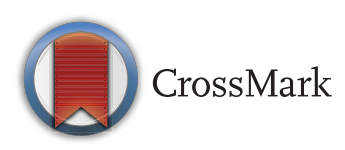

For numbered affiliations see end of article.

Correspondence to Dr losief Abraha; iosief_a@yahoo.it

\begin{abstract}
Introduction: Administrative healthcare databases are useful to investigate the epidemiology, health outcomes, quality indicators and healthcare utilisation concerning peptic ulcers and gastrointestinal bleeding, but the databases need to be validated in order to be a reliable source for research. The aim of this protocol is to perform the first systematic review of studies reporting the validation of International Classification of Diseases, 9th Revision and 10th version (ICD-9 and ICD-10) codes for peptic ulcer and upper gastrointestinal bleeding diagnoses.
\end{abstract}

Methods and analysis: MEDLINE, EMBASE, Web of Science and the Cochrane Library databases will be searched, using appropriate search strategies. We will include validation studies that used administrative data to identify peptic ulcer disease and upper gastrointestinal bleeding diagnoses or studies that evaluated the validity of peptic ulcer and upper gastrointestinal bleeding codes in administrative data. The following inclusion criteria will be used: (a) the presence of a reference standard case definition for the diseases of interest; (b) the presence of at least one test measure (eg, sensitivity, etc) and (c) the use of an administrative database as a source of data. Pairs of reviewers will independently abstract data using standardised forms and will evaluate quality using the checklist of the Standards for Reporting of Diagnostic Accuracy (STARD) criteria. This systematic review protocol has been produced in accordance with the Preferred Reporting Items for Systematic Reviews and Meta-Analysis Protocol (PRISMA-P) 2015 statement.

Ethics and dissemination: Ethics approval is not required given that this is a protocol for a systematic review. We will submit results of this study to a peerreviewed journal for publication. The results will serve as a guide for researchers validating administrative healthcare databases to determine appropriate case definitions for peptic ulcer disease and upper gastrointestinal bleeding, as well as to perform outcome research using administrative healthcare databases of these conditions.

Trial registration number: CRD42015029216.

\section{Strengths and limitations of this study}

- Validation of International Classification of Diseases, 9th Revision and 10th reversion (ICD-9 and ICD-10) diagnosis codes for peptic ulcer disease and upper gastrointestinal bleeding using administrative healthcare databases can contribute to health outcome research.

- This review will be the first to systematically identify and evaluate primary studies that validated the accuracy of ICD-9 and ICD-10 codes for peptic ulcer disease and upper gastrointestinal bleeding in administrative healthcare databases.

- The results from this systematic review will serve as a guide to determine appropriate case definitions for peptic ulcer and upper gastrointestinal bleeding.

- The main limitation is that validated diagnosis codes or algorithms are context-specific, and may not be generalisable to other settings.

\section{INTRODUCTION}

Non-variceal upper gastrointestinal bleeding (UGIB) is associated with significant morbidity and mortality. It has an incidence rate from 48 to 160 cases per 100000 per year, and greater incidences in men and older people. ${ }^{12}$ Although UGIB and peptic ulcer bleeding are diminishing in the general population, hospitalisation rates from ulcer complications are growing in older populations. ${ }^{3}$ The most frequent risk factors for non-variceal UGIB comprise Helicobacter pylori infection, and the use of NSAIDs/aspirin, and other antiplatelet and anticoagulant medications. (Up to $67 \%$ of cases of UGIB are caused by peptic ulcer disease (PUD)..$^{1}$ ) Both $H$. pylori infection and NSAIDs are independent risk factors for PUD and UGIB. $^{4}$ 
Health authorities generate and maintain large administrative healthcare databases that typically contain information and data regarding health resource utilisation (eg, hospitalisations, outpatient care and drug prescriptions) and vital statistics. ${ }^{5}$ For research, one of the advantages of administrative databases is that they passively collect data at a population level with longitudinal follow-up, making their results easily generalisable. In addition, they are considered to be cost-effective compared with primary data collection. ${ }^{6}{ }^{7}$ The main disadvantage of these databases is that they are generated for administrative purposes, such as billing, and as a repository for patient hospital records, and not for research, hence, the diagnostic codes for specific disorders must be validated according to an accepted 'gold standard' reference diagnosis. ${ }^{8-14}$

In the gastrointestinal field, administrative healthcare databases have been used to estimate the epidemiology of PUD ${ }^{15}$ and UGIB, ${ }^{16}$ to assess drug-related gastrointestinal outcomes, ${ }^{17-19}$ to conduct active drug surveillance $^{20}$ and health service quality evaluation. ${ }^{21}{ }^{22}$

Current administrative databases use the International Classification of Diseases, 9th Revision (ICD-9) or 10th Revision (ICD-10) codes for PUD and UGIB. Validation of diagnostic codes is of particular interest to national healthcare authorities to perform surveillance of medical products and epidemiological studies of diseases. For example, the US Food and Drug Administration has sponsored a pilot project, Mini-Sentinel, with the aim of performing active surveillance to improve safety signals that emerge for newly released medical products. To implement this work, the programme needed to identify algorithms used to detect a number of health outcomes of interest using administrative data sources and identify the performance characteristics of these algorithms. ${ }^{23}$ The Mini-Sentinel programme produced a series of systematic reviews of validated methods and case definitions, to identify various diseases or health outcomes in administrative data, including cardiocerebrovascular diseases ${ }^{24-28}$ and other conditions. ${ }^{29-33}$ For the purpose of establishing best practices in the use of administrative data for health research and surveillance, the Canadian Rheumatology Administrative Data Network conducted a systematic review of studies reporting on the validity of diagnostic codes to identify cardiovascular diseases. ${ }^{34-36}$ Likewise, the Regional Health Authority of Umbria, is interested in the validity of administrative data diagnoses and in identifying case definitions and the algorithms developed for different diseases, including cancer (breast, lung and colorectal) ${ }^{9}{ }^{11}$ chronic obstructive pulmonary disease ${ }^{13}$ and non-variceal UGIB, which is the focus of this article.

In the medical literature, at the present time, the validity and performance of algorithms employing diagnostic codes for PUD and UGIB have not been systematically investigated. With the current protocol, we plan to systematically evaluate validation studies of diagnostic codes corresponding to these gastrointestinal conditions in administrative databases.
METHODS

\section{Literature search}

Published peer-reviewed articles will be identified through comprehensive searches of MEDLINE, EMBASE, Web of Science and the Cochrane Library from their inception. We will use a search strategy that we developed based on the combination of: (a) keywords and Medical Subject Headings (MeSH) terms to identify records regarding PUD and UGIB; (b) terms to identify studies likely to contain validity or accuracy measures; and (c) a search strategy, based on the combination of terms used by Benchimol et $a \vec{l}^{37}$ and the Mini-Sentinel programme, ${ }^{38}{ }^{39}$ which is designed to accurately identify studies that use healthcare administrative databases. The search strategy is available as online supplementary appendix 1 . Relevant reference lists of key articles will be hand searched in order to retrieve additional articles. Pertinent articles that cited the article of interest, identified through the preceding search strategy, will be sought through the 'Cited-By' tools in PubMed and Google Scholar. Two independent reviewers will screen titles and abstracts for eligibility. Discussion will be used to resolve discrepancies.

This review protocol has been prepared according to the Preferred Reporting Items for Systematic reviews and Meta-Analysis Protocols (PRISMA-P) 2015 statement ${ }^{40}$ and the results will be presented following the PRISMA flow diagram (figure 1). ${ }^{41}$ This protocol has also been published in the PROSPERO International Prospective Register of Systematic Reviews with registration number CRD42015029216 (http://www.crd.york.ac. uk/PROSPERO).

\section{Inclusion criteria}

Type of studies

We will consider any type of diagnostic (cross-sectional, retrospective or prospective) cohort study, without limits in publication date, and published in English, for inclusion.

\section{Population}

The target populations will include patients of any age and sex with peptic ulcer or gastrointestinal haemorrhage. Since there are substantial differences between in-hospital and outpatient upper gastrointestinal bleeders in terms of both clinical risk profile and treatment patterns ${ }^{42}$ we will consider two types of cohorts with bleeding: (a) patients who have been admitted to a hospital due to non-variceal UGIB caused by peptic ulcer and (b) outpatients who have been visited for peptic ulcer or gastrointestinal bleeding.

\section{Index test}

Studies that validated diagnostic codes or algorithms related to ICD-9 or ICD-10 for PUD or UGIB will be considered. The ICD-9 codes for PUD and UGIB are: $531.0-531.7,531.9$ for gastric ulcers and haemorrhage, 532.0-532.7, 532.9 for duodenal ulcers and haemorrhage, 
Figure 1 Study screening process.

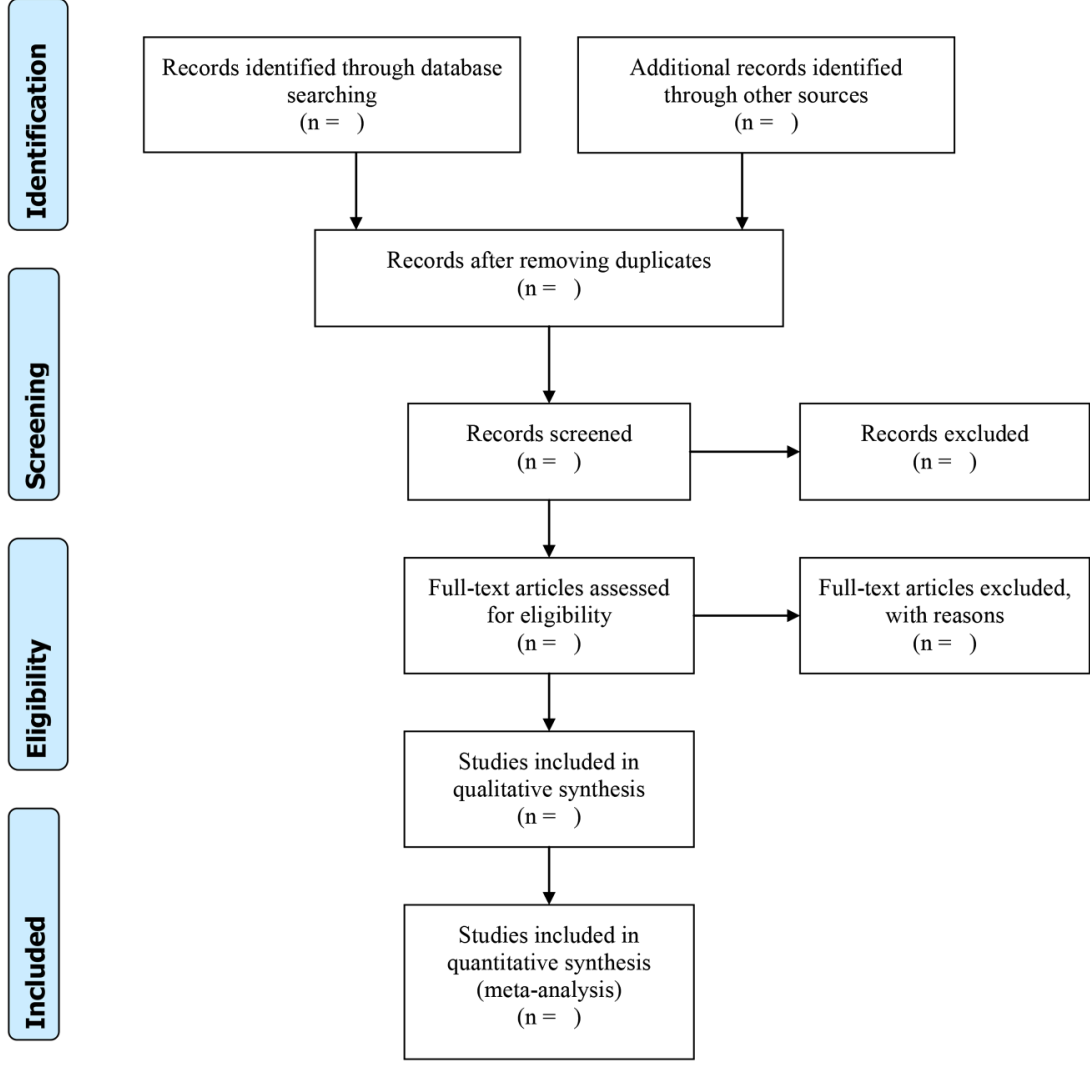

533.0-533.7, 533.9 for peptic ulcers and haemorrhage, $534.0-534.7,534.9$ for gastrojejunal ulcers and haemorrhage, and 578.0, 578.1, 578.9 for gastrointestinal haemorrhage. The ICD-10 codes are K25.0-K25.7 and K25.9 for gastric ulcers and haemorrhage, K26.0-K26.7 and K26.9 for duodenal ulcers and haemorrhage, K27.0-K27.7 and K27.9 for peptic ulcers and haemorrhage and K28.0-K28.7 and K28.9 for gastrojejunal ulcers and haemorrhage and K92.0, K92.1 and K92.2 for gastrointestinal haemorrhage. Detailed descriptions of each ICD code are reported in online supplementary appendix 2.

\section{Reference standard}

Studies will be considered in which the diagnoses of target diseases were confirmed through review of medical charts, medical notes or electronic health records. Confirmed peptic ulcers will include cases of active gastric or duodenal ulcers, or gastroduodenal perforation, as confirmed by surgery, endoscopy, X-ray or autopsy. Confirmed UGIB will include cases of haemorrhage from gastric or duodenal ulcers, haemorrhagic gastritis, duodenitis or gastroduodenal perforation, confirmed by surgery, endoscopy, X-ray or autopsy.

\section{Outcome}

Studies that reported the accuracy of administrative data codes to discriminate cases of PUD or UGIB, at least in terms of sensitivity or positive predictive values (PPVs) will be eligible for inclusion.

\section{Selection process}

During the initial stage, titles and abstracts will be screened to identify potentially eligible studies. Subsequently, full texts of articles will be obtained and evaluated to determine if they meet the inclusion and exclusion criteria. We will perform data abstraction with standardised data collection forms, which will be tested on a sample of eligible articles beforehand. Title and abstract screening, full-text screening and data abstraction will be carried out, independently, and in duplicate, by two review authors. Any discrepancies will be resolved by consensus, and where necessary, by involving a third review author. Calibration exercises will be performed at each step of the process.

\section{Data extraction}

Data extraction will include the following information:

I. The details of the included study (including title, year and journal of publication, country of origin, and sources of funding; the first author will be used as the study ID);

II. The disease of interest (peptic ulcer or UGIB);

III. The target population from which the administrative data were collected;

IV. The type of administrative database used (eg, hospitalisation discharge data), outpatient records (eg, physician billing claims), etc;

V. The ICD-9 or ICD-10 code used;

VI. External validation; 
VII. Use of training and testing cohorts;

VIII. The reference standard used to determine the validity of the diagnostic code (eg, medical chart review, patient self-reports, disease registry, etc);

IX. The characteristic of the test used to determine the validity of the diagnostic code or algorithm (eg, sensitivity, specificity, PPVs and negative predictive values (NPVs), area under the receiver operating characteristic (ROC) curve, likelihood ratios (LR) and $\kappa$-statistics);

$\mathrm{X}$. Any conflict of interest.

\section{Quality assessment}

The design and method of the included primary studies will be assessed using a checklist developed by Benchimol et $a l,{ }^{37}$ based on the criteria published by the Standards for Reporting of Diagnostic Accuracy (STARD) initiative for the accurate reporting of studies using diagnostic studies. ${ }^{43}$ The checklist is provided in online supplementary appendix 3 . The presence of potential biases within the studies will be reported descriptively.

No subgroup analysis or publication bias assessment is anticipated.

\section{Analysis}

For each algorithm, we will abstract the validation statistics provided in the included studies. Validation statistics may include sensitivity, specificity, PPV and NPV. We will calculate $95 \%$ CIs when they are not reported in the articles. Where sufficient and homogeneous data are available we will derive summary estimates of sensitivity and specificity and their 95\% CIs data using a bivariate model. ${ }^{44}$ Data will be meta-analysed using a randomeffects model so that sensitivity and specificity are assumed to vary across studies. Separate meta-analyses will be provided based on the administrative data source (outpatient vs inpatient data), type of ICD code (ICD-9 or ICD-10) and type of disease (ulcer or haemorrhage). We will perform subgroup analyses according to the timing of publication and ICD code assessed to examine whether accuracy data have changed overtime.

In addition, summary ROC curves will be constructed and pooled estimates of LR+, LR- and diagnostic OR will be calculated. Heterogeneity will be assessed by visual inspection of forest plots and ROC plots, as well as regression analysis suggested by Reitsma et al. ${ }^{44}$ Where there is important heterogeneity, we will not pool the data.

Publication bias will not evaluated, as the common tests available (Begg, Egger and Deeks tests) provide different results and thus are not interchangeable. ${ }^{45}$

\section{Ethics and dissemination}

Approval from an ethics committee is not required, since this review protocol will use publicly available data without directly involving human participants. An outline of the protocol has been published in the PROSPERO International Prospective Register of Systematic Reviews in 2015, registration number CRD42015029216. The results of the review will summarise the studies validating diagnostic codes that identify PUD and UGIB in administrative data. In addition, the results will serve as a guide to identify appropriate case definitions and algorithms of PUD and UGIB for researchers validating administrative healthcare databases, as well as for outcome research that uses administrative healthcare databases on these conditions. Findings of the review will be presented at relevant scientific conferences and disseminated through publication in a peer-reviewed journal.

\section{Author affiliations}

${ }^{1}$ Health Planning Service, Regional Health Authority of Umbria, Perugia, Italy ${ }^{2}$ Scientific Directorate, Italian National Research Center on Aging, Ancona, Italy

${ }^{3}$ Department of General Medicine, Azienda USL Umbria 1, Perugia, Italy

${ }^{4}$ Department of Geriatrics and Geriatric Emergency Care, Italian National Research Center on Aging, Ancona, Italy

${ }^{5}$ Department of Cardiology, University of Perugia School of Medicine, Perugia, Italy

Contributors IA, JMR, FC, MO and AM conceived the study. JMR, IA, MLL, $\mathrm{FC}, \mathrm{MO}, \mathrm{CC}, \mathrm{GA}$ and $\mathrm{AM}$ were responsible for designing the protocol. IA, GA, AM, MO, JMR and FC drafted the protocol manuscript. JMR, IA, FC and MO developed the search strategy. JMR, IA, MLL, FC, MO, CC, GA and AM critically revised the successive versions of the manuscript and approved the final version.

Funding This review protocol was funded by the Regional Health Authority of Umbria.

Disclaimer The study funder was not involved in the study design or the writing of the protocol.

Competing interests None declared.

Provenance and peer review Not commissioned; externally peer reviewed.

Data sharing statement All the results from the final version of the systematic review will be published.

Open Access This is an Open Access article distributed in accordance with the Creative Commons Attribution Non Commercial (CC BY-NC 4.0) license, which permits others to distribute, remix, adapt, build upon this work noncommercially, and license their derivative works on different terms, provided the original work is properly cited and the use is non-commercial. See: http:// creativecommons.org/licenses/by-nc/4.0/

\section{REFERENCES}

1. Tielleman T, Bujanda D, Cryer B. Epidemiology and risk factors for upper gastrointestinal bleeding. Gastrointest Endosc Clin N Am 2015;25:415-28.

2. Kurien M, Lobo AJ. Acute upper gastrointestinal bleeding. Clin Med (Lond) 2015;15:481-5.

3. Lanas A, García-Rodriguez LA, Polo-Tomás M, et al. The changing face of hospitalisation due to gastrointestinal bleeding and perforation. Aliment Pharmacol Ther 2011;33:585-91.

4. Papatheodoridis GV, Sougioultzis S, Archimandritis AJ. Effects of Helicobacter pylori and nonsteroidal anti-inflammatory drugs on peptic ulcer disease: a systematic review. Clin Gastroenterol Hepatol 2006;4:130-42.

5. Jutte DP, Roos LL, Brownell MD. Administrative record linkage as a tool for public health research. Annu Rev Public Health 2011;32:91-108.

6. Schneeweiss S, Avorn J. A review of uses of health care utilization databases for epidemiologic research on therapeutics. J Clin Epidemiol 2005;58:323-37.

7. Motheral BR, Fairman KA. The use of claims databases for outcomes research: rationale, challenges, and strategies. Clin Ther 1997;19:346-66. 
8. West SL, Strom BL, Poole C. Validity of pharmacoepidemiologic drug and diagnosis data. John Wiley \& Sons, Ltd, 2007.

9. Abraha I, Giovannini G, Serraino D, et al. Validity of breast, lung and colorectal cancer diagnoses in administrative databases: a systematic review protocol. BMJ Open 2016;6:e010409.

10. Abraha I, Montedori A, Eusebi P, et al. The current state of validation of administrative healthcare databases in Italy: a systematic review. Pharmacoepidemiol Drug Saf 2012;21:400.

11. Abraha I, Serraino D, Giovannini G, et al. Validity of ICD-9-CM codes for breast, lung, and colorectal cancers in three Italian administrative healthcare databases: a diagnostic accuracy study protocol. BMJ Open 2016;6:e010547.

12. Pisa F, Castellsague J, Drigo D, et al. Accuracy of International Classification of Diseases, 9th Revision, Clinical Modification codes for upper gastrointestinal complications varied by position and age: a validation study in a cohort of nonsteroidal anti-inflammatory drugs users in Friuli Venezia Giulia, Italy. Pharmacoepidemiol Drug Saf 2013;22:1195-204.

13. Rimland JM, Abraha I, Luchetta ML, et al. Validation of chronic obstructive pulmonary disease (COPD) diagnoses in healthcare databases: a systematic review protocol. BMJ Open 2016;6: e011777.

14. Abraha I, Orso M, Grilli $\mathrm{P}$, et al. The current state of validation of administrative healthcare databases in Italy: a systematic review. Int J Stat Med Res 2014;3:309-20.

15. Thorsen K, Søreide JA, Kvaløy JT, et al. Epidemiology of perforated peptic ulcer: age- and gender-adjusted analysis of incidence and mortality. World J Gastroenterol 2013;19:347-54

16. Abougergi MS, Travis AC, Saltzman JR. The in-hospital mortality rate for upper $\mathrm{Gl}$ haemorrhage has decreased over 2 decades in the United States: a nationwide analysis. Gastrointest Endosc 2015;81:882-8.e1.

17. Chang HY, Zhou M, Tang W, et al. Risk of gastrointestinal bleeding associated with oral anticoagulants: population based retrospective cohort study. BMJ 2015;350:h1585.

18. De Berardis G, Lucisano G, D'Ettorre A, et al. Association of aspirin use with major bleeding in patients with and without diabetes. JAMA 2012;307:2286-94.

19. Jun M, James MT, Manns BJ, et al. The association between kidney function and major bleeding in older adults with atrial fibrillation starting warfarin treatment: population based observational study. BMJ 2015;350:h246

20. Coloma PM, Trifirò G, Schuemie MJ, et al. Electronic healthcare databases for active drug safety surveillance: is there enough leverage? Pharmacoepidemiol Drug Saf 2012;21:611-21.

21. Mattke S, Needleman J, Buerhaus $\mathrm{P}$, et al. Evaluating the role of patient sample definitions for quality indicators sensitive to nurse staffing patterns. Med Care 2004;42:II21-33.

22. Needleman J, Buerhaus $P$, Mattke $S$, et al. Nurse-staffing levels and the quality of care in hospitals. N Engl J Med 2002;346:1715-22.

23. Platt R, Carnahan RM, Brown JS, et al. The U.S. Food and Drug Administration's Mini-Sentinel program: status and direction. Pharmacoepidemiol Drug Saf 2012;21(Suppl 1):1-8.

24. Andrade SE, Harrold LR, Tjia J, et al. A systematic review of validated methods for identifying cerebrovascular accident or transient ischemic attack using administrative data. Pharmacoepidemiol Drug Saf 2012;21(Suppl 1):100-28.

25. Jensen PN, Johnson K, Floyd J, et al. A systematic review of validated methods for identifying atrial fibrillation using administrative data. Pharmacoepidemiol Drug Saf 2012;21(Suppl 1):141-7.

26. Saczynski JS, Andrade SE, Harrold LR, et al. A systematic review of validated methods for identifying heart failure using administrative data. Pharmacoepidemiol Drug Saf 2012;21(Suppl 1):129-40.
27. Tamariz L, Harkins T, Nair V. A systematic review of validated methods for identifying venous thromboembolism using administrative and claims data. Pharmacoepidemiol Drug Saf 2012;21(Suppl 1):154-62.

28. Tamariz L, Harkins T, Nair V. A systematic review of validated methods for identifying ventricular arrhythmias using administrative and claims data. Pharmacoepidemiol Drug Saf 2012;21(Suppl 1):148-53.

29. Chung CP, Rohan P, Krishnaswami S, et al. A systematic review of validated methods for identifying patients with rheumatoid arthritis using administrative or claims data. Vaccine 2013;31(Suppl 10): K41-61.

30. Idowu RT, Carnahan R, Sathe NA, et al. A systematic review of validated methods to capture myopericarditis using administrative or claims data. Vaccine 2013;31(Suppl 10):K34-40.

31. Moores KG, Sathe NA. A systematic review of validated methods for identifying systemic lupus erythematosus (SLE) using administrative or claims data. Vaccine 2013;31(Suppl 10):K62-73.

32. Williams SE, Carnahan R, Krishnaswami S, et al. A systematic review of validated methods for identifying transverse myelitis using administrative or claims data. Vaccine 2013;31(Suppl 10):K83-7.

33. Williams SE, Carnahan R, McPheeters ML. A systematic review of validated methods for identifying uveitis using administrative or claims data. Vaccine 2013;31(Suppl 10):K88-97.

34. McCormick N, Bhole V, Lacaille D, et al. Validity of diagnostic codes for acute stroke in administrative databases: a systematic review. PLOS ONE 2015;10:e0135834

35. McCormick N, Lacaille $\mathrm{D}$, Bhole $\mathrm{V}$, et al. Validity of heart failure diagnoses in administrative databases: a systematic review and meta-analysis. PLOS ONE 2014;9:e104519.

36. McCormick N, Lacaille $\mathrm{D}$, Bhole $\mathrm{V}$, et al. Validity of myocardial infarction diagnoses in administrative databases: a systematic review. PLOS ONE 2014;9:e92286.

37. Benchimol El, Manuel DG, To T, et al. Development and use of reporting guidelines for assessing the quality of validation studies of health administrative data. J Clin Epidemiol 2011;64:821-9.

38. Carnahan RM, Moores KG. Mini-Sentinel's systematic reviews of validated methods for identifying health outcomes using administrative and claims data: methods and lessons learned. Pharmacoepidemiol Drug Saf 2012;21(Suppl 1):82-9.

39. McPheeters ML, Sathe NA, Jerome RN, et al. Methods for systematic reviews of administrative database studies capturing health outcomes of interest. Vaccine 2013;31(Suppl 10):K2-6.

40. Liberati A, Altman DG, Tetzlaff $\mathrm{J}$, et al. The PRISMA statement for reporting systematic reviews and meta-analyses of studies that evaluate healthcare interventions: explanation and elaboration. BMJ 2009;339:b2700.

41. Shamseer L, Moher D, Clarke M, et al. Preferred reporting items for systematic review and meta-analysis protocols (PRISMA-P) 2015: elaboration and explanation. BMJ 2015;349:g7647.

42. Marmo R, Koch M, Cipolletta L, et al. Predicting mortality in patients with in-hospital nonvariceal upper GI bleeding: a prospective, multicenter database study. Gastrointest Endosc 2014;79:741-49. e1

43. Bossuyt PM, Reitsma JB, Bruns DE, et al. Towards complete and accurate reporting of studies of diagnostic accuracy: the STARD initiative. Ann Intern Med 2003;138:40-4.

44. Reitsma JB, Glas AS, Rutjes AW, et al. Bivariate analysis of sensitivity and specificity produces informative summary measures in diagnostic reviews. J Clin Epidemiol 2005;58:982-90.

45. van Enst WA, Ochodo E, Scholten RJ, et al. Investigation of publication bias in meta-analyses of diagnostic test accuracy: a meta-epidemiological study. BMC Med Res Methodol 2014;14:70. 